\title{
EMAP-II Expression Is Associated with Macrophage Accumulation in Primary Uveal Melanoma
}

\author{
Ruud Clarijs, Lia Schalkwijk, Dirk J. Ruiter, and Robert M. W. de Waal
}

Purpose. Primary uveal melanoma may contain arcs, loops, and networks of periodic acid-Schiff (PAS)-positive patterns, along which numerous macrophages are present. Their recruitment into tumor tissue is mediated by chemotactic cytokines, for which vascular endothelial growth factor (VEGF)-C and endothelial monocyte-activating polypeptide ((EMAP)-II are candidates. In this study, the extent of VEGF-C and EMAP-II immunoreaction was related to infiltration of macrophages.

MeTHods. Serial sections of 25 primary uveal melanoma lesions were analyzed by immunohistochemistry.

Results. The analysis showed no correlation of VEGF-C immunoreaction and localization of macrophages. However, accumulation of macrophages occurred at sites of EMAP-II expression, especially in areas containing nests of tumor cells, surrounded by arcs, loops, and network patterns. In tumors with a strong EMAP-II immunoreaction, the adhesion molecule intracellular adhesion molecule (ICAM)-1 was strongly expressed on endothelial cells. EMAP-II-positive endothelial cells did not express VEGF receptor-2. However, extensive release of von Willebrand factor was observed. Signs of apoptosis were found neither in tumor cells nor endothelial cells.

Conclusions. In uveal melanoma, macrophages accumulate at sites of EMAP-II expression. Based on the results, it may be hypothesized that this process of chemotaxis is facilitated by EMAP-II- dependent expression of ICAM-1 on vascular endothelial cells and concomitantly leads to localized vascular damage, as indicated by release of von Willebrand factor. (Invest Ophthalmol Vis Sci. 2003;44:1801-1806) DOI:10.1167/ iovs.02-0624

$I_{\mathrm{e}}^{\mathrm{n}}$ both primary uveal and cutaneous melanoma, nine different patterns of extracellular matrix deposition have been identified by conventional periodic acid-Schiff (PAS) staining. ${ }^{1-3}$ These different patterns appear to be of prognostic significance. Especially, the presence of PAS-positive arcs, loops, and network patterns has been associated with poor survival. ${ }^{1-4}$

In a recent study, ${ }^{5}$ we suggested that these fibrovascular septa constitute a fluid-conducting meshwork and contain endothelial cells, stromal cells, and macrophages along the arcs, loops, and network patterns. Other reports ${ }^{6,7}$ also have noted the abundant presence of macrophages in uveal melanoma. Despite this abundance, ${ }^{5-7}$ their role in uveal melanoma is largely unknown. Macrophages may be involved in angiogen-

From the Department of Pathology, University Medical Centre, Nijmegen, The Netherlands.

Supported by Grant 98-1816 from the Dutch Cancer Society.

Submitted for publication June 25, 2002; revised October 25, 2002; accepted November 23, 2002.

Disclosure: R. Clarijs, None; L. Schalkwijk, None; D.J. Ruiter, None; R.M.W. de Waal, None

The publication costs of this article were defrayed in part by page charge payment. This article must therefore be marked "advertisement" in accordance with 18 U.S.C. $\$ 1734$ solely to indicate this fact.

Corresponding author: Ruud Clarijs, Department of Pathology, University Medical Centre, PO Box 9101, 6500 HB Nijmegen, The Netherlands; r.clarij@@pathol.umcn.nl. esis (reviewed in Ref. 8), which is supported by a recent study that showed that the number of macrophages was related to microvascular density. ${ }^{6}$ High numbers of macrophages located in the tumor have also been related to poor prognosis in primary uveal melanoma ${ }^{6,7}$ and in other tumor types. ${ }^{9-11}$ Furthermore, their strict colocalization with the arcs, loops, and network patterns ${ }^{5,6}$ suggests either a role in the development of these patterns or, alternatively, the use of these patterns to invade the tumor lesion. It is unknown how invasion of macrophages in uveal melanoma is mediated. As recently reported, in xenografted melanoma, macrophages were recruited by vascular endothelial growth factor (VEGF)-C, ${ }^{12}$ which is also expressed in uveal melanoma. ${ }^{13}$

Besides VEGF-C, endothelial monocyte-activating polypeptide (EMAP)-II may also be involved in the process of macrophage invasion because of its ability to attract monocytes and granulocytes. ${ }^{14,15}$ EMAP-II mRNA has been detected in normal tissue and in tumors. ${ }^{16-21}$ It was originally described as a tumor-derived cytokine, isolated from the MethA tumor supernatant. ${ }^{14,15}$ It is involved in embryonic development, ${ }^{16,22,23}$ inflammation, ${ }^{21,24}$ and autoimmune disease. ${ }^{25}$ The possible effects of EMAP-II on tumor growth are complex. ${ }^{26}$ On the one hand, release of EMAP-II by hypoxic or apoptotic tumor cells may be involved in macrophage influx and subsequent macrophage-mediated angiogenesis in hypoxic or apoptotic areas. ${ }^{14,15,17}$ Indeed, hypoxic prostate adenocarcinoma cells release EMAP-II. ${ }^{20}$ Infiltration of blood-stream monocytes in tumor tissue is mediated by endothelial adhesion proteins ICAM-1 and VCAM-1 and, to a lesser extent, P- and E-selectin (for extensive review, see Ref. 27). If EMAP-II expression mediates recruitment of monocytes and resident macrophages to malignant tumors, this would require concomitant expression of endothelial adhesion proteins. It was already described previously that $\mathrm{P}$ - and E-selectin are upregulated on endothelial cells under the influence of recombinant EMAP-II in vitro. ${ }^{14}$ On the other hand, EMAP-II-mediated influx of macrophages may enhance the immune response against a tumor and thereby counteract tumor growth. Furthermore, EMAP-II may also inhibit tumor growth by targeting the tumor vascular bed through induction of endothelial cell apoptosis, ${ }^{18}$ by binding to $\alpha$-adenosine triphosphate (ATP) synthase ${ }^{28}$ and priming the tumor vasculature for (local) destruction by TNF- $\alpha .{ }^{19,29,30} \mathrm{Ev}$ idence of these previously proposed mechanisms is circumstantial, however, and therefore speculative. Most data in recent literature were obtained by studying the effects of applied, recombinant EMAP-II. Thus, the effects of endogenous EMAP-II produced in the tumor interstitium are unclear.

To elucidate the role of endogenous, tumor-derived EMAP-II in primary uveal melanoma, we evaluated both the occurrence and localization of EMAP-II, ICAM-1, and VCAM-1 and determined the presence of macrophages by immunohistochemistry. In addition, levels of apoptosis, endothelial activation (as indicated by expression of VEGF receptor (VEGFR)- $2^{31}$ and signs of endothelial damage (as characterized by the release of von Willebrand Factor $[\mathrm{vWF}]^{32}$ ) were studied. Finally, we investigated VEGF-C expression in relation to the presence and localization of macrophages. 


\section{Materials ANd Methods}

\section{Tissues}

Tissues were obtained according to guidelines of the Dutch legislation, and our work adhered to the tenets of the Declaration of Helsinki. Frozen specimens from 25 uveal melanomas were obtained from the disease archives of the University Medical Centre Nijmegen where they were stored at $-130^{\circ} \mathrm{C}$. Presence of disease in all specimens had been determined by a pathologist. All primary melanomas were obtained by surgery, and data of the clinical outcome were obtained for 16 patients with a follow-up time from 1 to 13 years (median 4.5 years). The uveal melanomas varied from 7 to $28 \mathrm{~mm}$ in diameter (median, $22 \mathrm{~mm}$ ) and included 23 choroidal and 2 ciliary melanoma lesions. Uveal melanoma lesions were divided in two parts along the maximal diameter. One part was formalin fixed and the other part was snap frozen. Hematoxylin and eosin (H\&E) staining was used on paraffin-embedded sections, and the 25 uveal melanomas were classified as 5 spindle cell type and 20 epithelioid or mixed type. Azan and PAS histochemistry without counterstaining on unbleached paraffin-embedded sections showed that 10 (44\%) uveal melanomas contained loops and networks matrix patterns (Fig. 1A). ${ }^{1,2,5,13,33}$ It appeared that in lesions previously classified as arcs, loops, and network patterns, ${ }^{1,2}$ the presence of arcs (with and without branching) was minimal. In addition, the arcs, loops, and network patterns were focally present in a tumor. Therefore, it is possible that sampling errors occurred, leading to evaluation of a section containing no network patterns, whereas they were present in other parts of the tumor. Taken together, these considerations may explain the relatively low incidence of arcs, loops, and network patterns in our specimens. In our experience, ${ }^{5,13}$ the extent of melanin pigmentation did not significantly hamper the detection of extracellular matrix by PAS and Azan histochemistry. The use of PAS and Azan histochemistry in identifying matrix patterns was recently confirmed by immunofluorescence and electron microscopy. ${ }^{5}$

\section{Antibodies}

Antibodies used for immunohistochemistry are listed in Table 1. AntiCD34 (QBEnd/10), anti-CD68 (Kp1 and PG-M1), and anti-vWF were from Dako (Glostrup, Denmark). Anti-ICAM-1 (PN-E12.1) was raised in our laboratory, ${ }^{35}$ and anti-VCAM-1 was from Immunotech SA (Marseilles, France). Anti-VEGFR-2 (Clone KDR-2) was from Sigma (St. Louis, MO). Goat polyclonal antibodies to VEGF-C were purchased from Santa Cruz Biotechnology (Santa Cruz, CA). Rabbit polyclonal antibodies EMAP-II (SA 2846) were obtained from Matthias Clauss (Max Planck Institute, Bad Nauheim, Germany). The rabbit was boosted once against a purified recombinant peptide containing 20 amino acids located at the amino terminal site of the mature human EMAP-II form. Control experiments demonstrated the specificity of the antibodies to both mouse and human EMAP-II and its precursor p43, as previously shown. ${ }^{17,34}$

\section{Immunohistochemistry}

Four-micrometer cryosections were air dried and fixed in acetone at room temperature for 10 minutes. Only strongly pigmented sections were bleached by incubating the sections in $3.0 \%$ ( $\mathrm{vol} / \mathrm{vol}$ ) hydrogen peroxide and $1.0 \%(\mathrm{wt} / \mathrm{vol})$ disodium hydrogen phosphate for 18 hours at room temperature. Cryosections were incubated with $3 \%$ hydrogen peroxide for 30 minutes and subsequently with $20 \%$ normal goat (EMAP-II and vWF staining) or horse serum (other staining) for 10 minutes. Successive sections of each specimen were incubated with QBEnd/10 (diluted 1:100), anti-EMAP-II (diluted 1:80) polyclonal antibodies, Kp1 (diluted 1:100), PG-M1 (diluted 1:50), PN-E12.1 (undiluted supernatant), anti-VCAM-1 (diluted 1:40), anti-vWF (diluted 1:300), or anti-VEGF-C (diluted 1:20) for 60 minutes at room temperature. AntiVEGFR-2 (diluted 1:400) was incubated overnight at $4^{\circ} \mathrm{C}$. After the first and all following incubation steps, sections were rinsed with ample phosphate-buffered saline (PBS). Then secondary, 1:200 diluted biotinylated affinity-purified anti-rabbit IgG (for the EMAP-II and vWF stain-
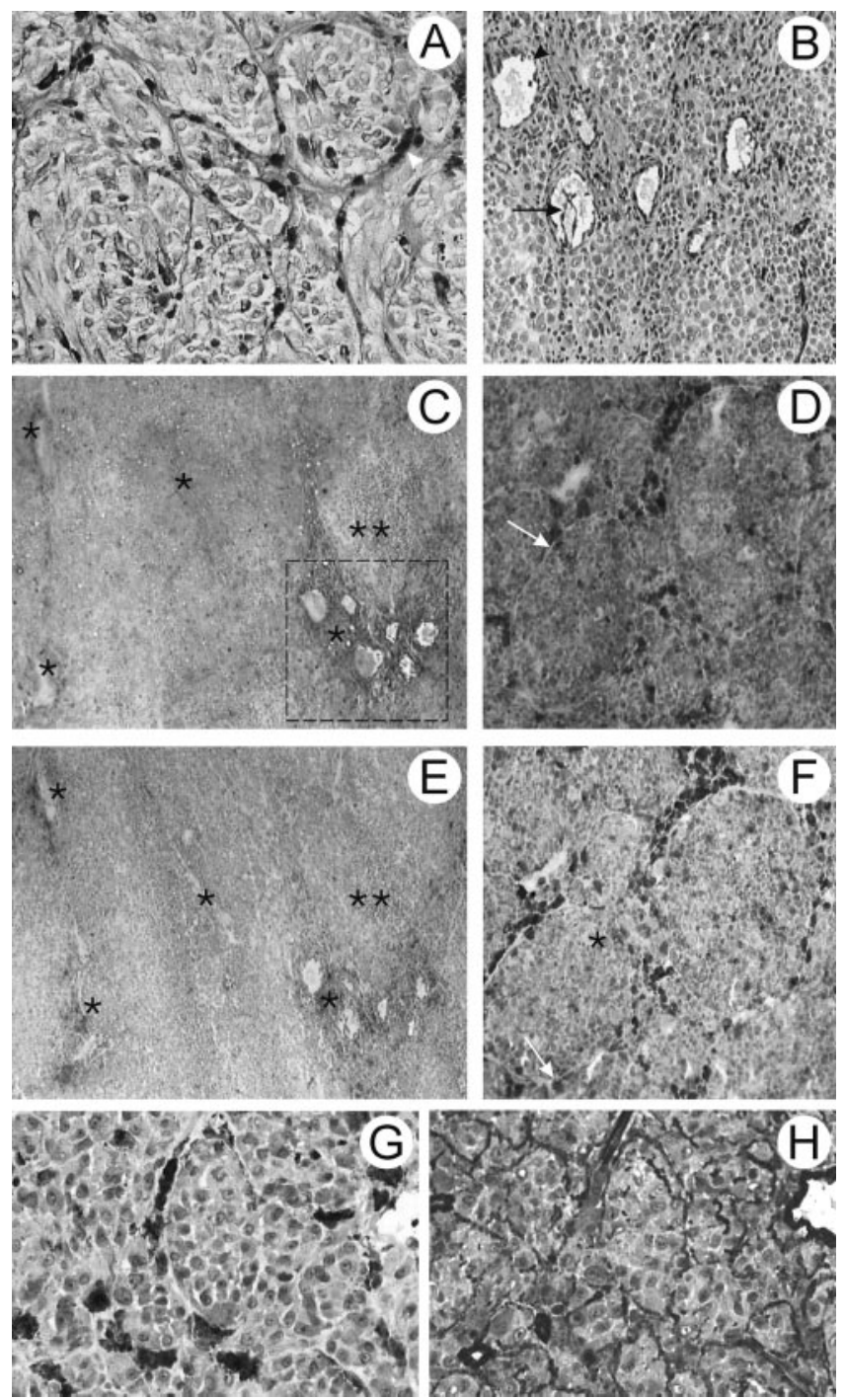

FIGURE 1. PAS-histochemistry (A) and immunohistochemical analysis of CD34 (B) and EMAP-II (C, D) expression and presence of macrophages (i.e., CD68-positive cells) (E, F) KP1, (G) MG-P1, and laminin (H) in (serial) sections of primary uveal melanoma. PAS histochemistry without bleaching decorates loops and network patterns containing melanophages (A, arrowbead). The vasculature was identified by an anti-CD34 mAb (B). Often the endothelial lining appeared to be discontinuous (arrowbead) and damaged (arrow). In areas with strongest EMAP-II immunoreaction (C), abundant CD68 staining was present, associated with the vasculature (CD34 staining of the boxed area is depicted in $\mathbf{B})$, indicating the accumulation of macrophages $(\mathbf{E})$. (C, E, *) Corresponding areas. EMAP-II positivity of tumor cells in an area without accumulation of macrophages can be explained by the detection of its precursor $\mathrm{p} 43$ which is retained intracellularly. ${ }^{17,34}$ EMAP-II positivity of tumor cells in an area without accumulation of macrophages is explained by the detection of the EMAP-II 34,000 form, which is retained intracellularly. ${ }^{17,34}$ Inflammatory infiltrating cells (other than macrophages) are negative for EMAP-II (C, ***). Especially in melanoma-containing loops and network patterns (D, arrow), EMAP-II immunoreaction was high. Next to macrophages (F, arrow), tumor cells were occasionally positive for CD68, stained by the KP1 mAb (F, *). In a serial section, macrophages stained for CD68 by MP-G1 mAb (G) showed that they are closely related to the extracellular matrix structures containing laminin $(\mathbf{G}, \mathbf{H})$. Use of CD68 mAb Kp1 and MG-P1 confirmed earlier data of Mäkitie et al. ${ }^{6}$ (B-H) Counterstained with Harris' hematoxylin. Magnification $(\mathbf{A}, \mathbf{B}, \mathbf{D}, \mathbf{F}) \times 100$; $(\mathbf{C}, \mathbf{E}) \times 40 ;(\mathbf{G}, \mathbf{H}) \times 400$. 
TABLE 1. Primary Antibodies Used: Antigens and Type of Cells Stained

\begin{tabular}{lll}
\hline Antigen & Antibody & \multicolumn{1}{c}{ Type of Cell } \\
\hline CD34 & QBEnd/10 & Endothelial \\
EMAP-II & Anti-EMAP-II* & Tumor, endothelial \\
CD68 & Kp1 & Macrophages \\
CD68 & PG-M1 & Macrophages \\
VEGF-C & Anti-VEGF-C* & Macrophages \\
ICAM-1 & PN-E12.1 & Endothelial \\
VCAM & anti-VCAM-1 & Endothelial \\
vWF & anti-vWF* & Endothelial \\
VEGFR-2 & anti-VEGFR-2 & Endothelial \\
\hline
\end{tabular}

${ }^{*}$ Polyclonal antibody.

ing), anti-goat (for VEGF-C staining), or anti-mouse IgG (all other staining) (Vectastain; Vector Laboratories, Burlingame, CA) was incubated for 30 minutes, followed by a 30-minute (VEGFR-2 and VEGF-C staining) or 45-minute (all other staining) incubation with peroxidaselabeled avidin-biotin complex (ABC, Vectastain; Vector Laboratories). Subsequently, the sections stained with VEGFR-2 and VEGF-C antibodies were incubated for 10 minutes with biotinylated tyramine (dilution 1:200), followed by a 20-minute incubation with ABC-peroxidase solution (catalyzed reporter deposition method ${ }^{36}$ ). All staining was developed by a 10 -minute incubation with a $0.4 \mathrm{mg} / \mathrm{mL}$ 3-amino-9-ethylcarbazole solution (Aldrich, Steinheim, Germany).

Four-micrometer paraffin-embedded serial sections were deparaffinized in xylene and rehydrated in an ethanol series. Antigen retrieval was performed by boiling sections for 10 minutes in sodium citrate buffer ( $\mathrm{pH}$ 6.0) using a microwave oven. Endogenous peroxidase activity was blocked by treating the sections for 30 minutes with PBS containing 3\% hydrogen peroxide. Then, sections were incubated with $20 \%$ normal horse serum (Vector Laboratories) for 10 minutes. Of each specimen, the sections directly adjacent to the section stained by Azan histochemistry were incubated with QBEnd/10 mAb (diluted 1:50) and further processed as described earlier.

In control sections, primary antibodies were omitted. All staining was counterstained for 45 seconds with Harris' hematoxylin (Merck, Darmstadt, Germany) at room temperature and were mounted (Imsol; Klinipath BV, Duiven, The Netherlands).

\section{Determination of Macrophage Density}

In a subset of tumors expressing EMAP-II, the density of macrophages in areas with low or without immunoreaction and in areas with strong immunoreaction was assessed by visual examination of EMAP-II and CD68 immunostaining, using an ocular with counting grid at $163 \times$ magnification. In a tumor containing areas with both low and strong EMAP-II immunoreaction, numbers of macrophages were counted in 10 nonoverlapping fields for either level of immunoreaction. Statistical analysis was performed by a paired $t$-test.

\section{Detection of Apoptosis}

Apoptotic cells were detected in $4-\mu \mathrm{m}$ cryosections with a cell viability test (ApopTag Kit S7100; Introgen Therapeutics, Houston, TX), according to the manufacturer's guidelines.

\section{Results}

\section{Vascular Staining}

In all 25 uveal melanomas, the vasculature was identified by anti-CD34 mAb staining on frozen sections (Table 1) and morphologic properties, as described previously. 5,13,37 Often, the endothelial layer was discontinuous, compatible with endothelial damage (Fig. 1B). Although blood vessel density varied among different lesions, no evident increase of blood vessel density in distinct areas within a tumor lesion was observed. Azan and PAS histochemistry (Fig. 1A) and CD34-immunohistochemistry of serial paraffin-embedded sections indicated that the blood vasculature was closely associated with loops and network patterns, as described previously. ${ }^{5}$

\section{Colocalization of EMAP-II Expression and Macrophages}

EMAP-II expression was detected in 23 (92\%) of 25 uveal melanomas as a cytoplasmic staining of tumor cells (Figs. 1C, 1D). In 15 tumors, EMAP-II expression was localized in circumscript areas. Especially, tumor cells directly surrounding blood vessels were strongly positive for EMAP-II. In eight melanomas, all tumor cells were intensely positive for EMAP-II (referred to as ubiquitous immunoreaction). By comparison to parallel endothelial CD34 staining in serial sections, EMAP-II was also present on blood vascular endothelium in three tumors in the areas with local expression of EMAP-II, whereas in six tumors with ubiquitous EMAP-II staining of tumor cells, endothelial cells were also ubiquitously positive. Especially in areas of loops and network patterns, all tumor cells showed strong EMAP-II immunoreaction (Fig. 1D). Strong endothelial EMAP-II immunoreaction was also observed in areas with necrosis (not shown). Finally, background staining patterns that could interfere with EMAP-II expression analysis were not observed when using other polyclonal antibodies (e.g., anti-vWF polyclonal antibodies).

In all 25 melanomas, macrophages could be detected by CD68 staining and by morphologic characteristics (compare serial sections in Figs. 1E, 1F). When using Kp1 antibody, tumor cells were also weakly positive for CD68 in eight melanoma lesions (Fig. 1F), whereas incubation with the PG-M1 $\mathrm{mAb}$ did not result in CD68 staining of melanoma cells (Fig. $1 \mathrm{H})$. These data confirm earlier results. ${ }^{6,38}$ Both macrophages and melanophages were strongly associated with the extracellular loops and network patterns, which contain laminin (Figs. $1 \mathrm{G}, 1 \mathrm{H}) \mathrm{s}^{5}$

In the 15 tumors with local differences in EMAP-II staining intensity, macrophages were consistently more abundant in areas with higher EMAP-II levels, especially around blood vessels (compare serial sections in Figs. 1C, 1E). Counting of the number of macrophages in four of these tumors demonstrated a significant difference $(P<0.0001)$ between those in tumor areas with negative to low EMAP-II immunoreaction $(72 \pm 49$ macrophages $/ \mathrm{mm}^{2}$; mean $\pm \mathrm{SD}$ ) and those in areas with strong EMAP-II immunoreaction (457 \pm 129 macrophages $/ \mathrm{mm}^{2}$ ). Macrophages were detected around both EMAP-II-negative $(n=12)$ and -positive $(n=3)$ blood vessel endothelium. In eight melanomas with ubiquitous EMAP-II immunoreaction, macrophages were equally distributed over the tumor tissue. In these tumors, macrophages were present around EMAP-IInegative and -positive $(n=7)$ vessels as well. In areas containing loops and network extracellular matrix patterns, nests of tumor cells were intensively positive for EMAP-II immunostaining. In addition, EMAP-II immunoreaction was lower in areas containing no loops and network patterns compared with areas with these patterns within one lesion.

\section{ICAM-1 and VCAM-1 Immunostaining}

Coexpression of ICAM-1 and EMAP-II was found on tumor cells in all 23 EMAP-II-positive cases (Figs. 2A, 2B). Conversely, in areas with no or low tumoral EMAP-II immunoreaction, ICAM-1 was not detected on tumor cells. In only 3 of 15 tumors with local EMAP-II expression was ICAM-1 expression observed on EMAP-II-positive endothelium, whereas this was the case in 6 of 8 tumors (Figs. 2A, 2B) with generalized immunoreaction. ICAM-1 was often expressed on a subset of endothelial cells 

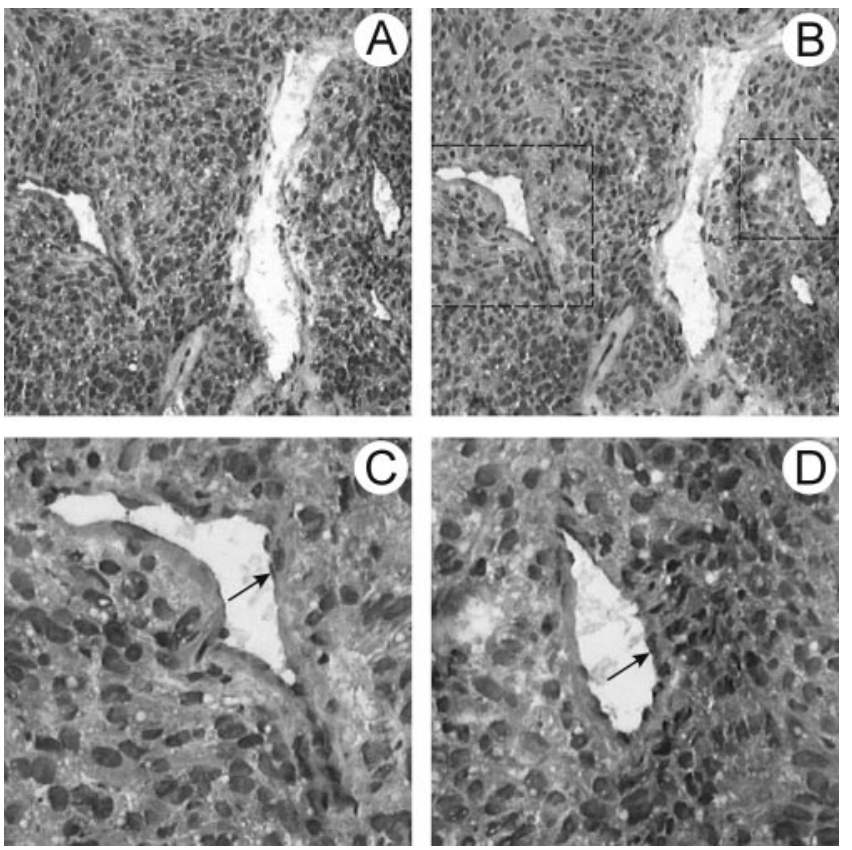

FIGURE 2. Immunohistochemical analysis in serial sections of EMAP-II (A) and ICAM-1 (B) expression in primary uveal melanoma. Intense ubiquitous EMAP-II staining and ICAM-1 expression were observed in both tumor and endothelial cells $(\mathbf{A}, \mathbf{B})$. Boxed areas in (B) are shown enlarged in (C) and (D). Part of the EMAP-II-positive endothelial cells express ICAM-1 (C, D, arrow) whereas the central EMAP-II-negative vessel expressed no ICAM-1 (A, B). All stains were counterstained with Harris' hematoxylin. Magnification: $(\mathbf{A}, \mathbf{B}) \times 100$; (C, D) $\times 250$.

(Figs. 2C, 2D). In this group of tumors, EMAP-II-negative endothelium did not express ICAM-1 (central vessel in Figs. 2A, 2B).

In only four melanomas, delicate VCAM-1 staining was detected on both EMAP-II-positive and -negative blood vascular endothelium (not shown). VCAM-1 expression was never observed on tumor cells.

\section{VEGFR-2 and vWF Immunostaining}

We analyzed 16 EMAP-II-positive uveal melanomas for the presence of VEGFR-2 and VWF as markers of endothelial activation and damage, respectively. In 14 melanomas, comparison to parallel EMAP-II staining demonstrated that EMAP-II-positive endothelial cells showed no expression of VEGFR-2 (Figs. 3A, 3B). However, strong staining of $\mathrm{VWF}$ in endothelial cells and underlying tissue indicated a release of VWF by this endothelium (Figs. 3A, 3C). Conversely, EMAP-II-negative endothelial cells expressed VEGFR-2 in five melanomas (Figs. 3D, 3E) whereas no evident damage was detected in these cells by vWF staining (not shown). In only two tumors, a weak colocalization of EMAP-II positivity, VEGFR-2, and absence of vWF-release was observed. Patterns similar to those of EMAP-II staining were not observed when using other polyclonal antibodies (e.g., anti-vWF polyclonal antibodies).

\section{Detection of Apoptosis}

Apoptosis was detectable in only 3 of 15 uveal melanomas. In none of the tumors were apoptotic endothelial cells found. In one tumor, an evidently necrotic area positive for EMAP-II, showed staining, whereas in the remaining tumor cells, apoptosis was absent. In the other two tumors, apoptosis was confined to a few tumor cells dispersed over the entire tumor area (not shown).

\section{Absence of Colocalization of VEGF-C Expression and Macrophages}

Finally, we analyzed 12 melanomas for expression of VEGF-C. In six melanomas, VEGF-C was expressed in restricted areas. No evident colocalization of accumulation or absence of macrophages and VEGF-C expression was observed in any lesion (data not shown).

\section{Correlation with Clinical Outcome}

In only one patient, liver metastasis was found 2 years after enucleation. This tumor contained PAS-positive loops and network patterns and ubiquitous EMAP-II immunoreaction of tumor and endothelial cells and macrophages was equally distributed over the lesion. In addition, ICAM-1 was expressed by the tumor and a subset of endothelial cells. No VCAM-1 expression or apoptosis was observed. EMAP-II-positive endothelial cells lacked expression of VEGFR-2 but stained strongly for vWF. VEGF-C expression was not evaluated.

\section{Discussion}

Recently, we described that the arcs, loops, and network extracellular matrix patterns may represent a fluid-conducting meshwork in xenografted and primary uveal melanoma. ${ }^{5}$ Our findings indicated that these structures closely parallel the previously described vascular channels. ${ }^{39}$ Although it has been demonstrated that melanoma cells may express nonmelanocytic markers like endothelial-cell-associated CD34 and macrophage-associated $\mathrm{CD} 68,{ }^{39,40}$ we could not confirm the presence of blood-conducting channels lined by tumor cells. Instead, septa consisting of extracellular matrix components that could be visualized by PAS and Azan histochemistry surrounded tumor cell nests. Remarkably, many macrophages (i.e., CD68-positive cells) were found associated with this meshwork in melanoma xenografts. We now have studied the presence and localization of macrophages in uveal melanoma, focusing on the mechanisms underlying infiltration by this cell
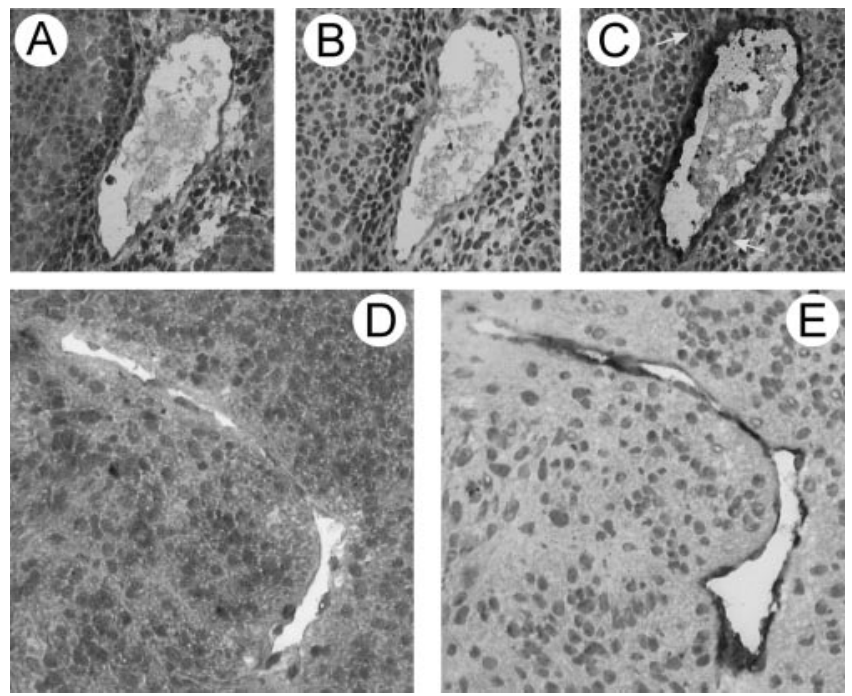

FIGURE 3. Immunohistochemical analysis in serial sections of EMAP-II (A, D), VEGFR-2 (B, E), and vWF (C) expression in primary uveal melanoma. Although endothelial EMAP-II immunoreaction was slight (A), VEGFR-2 were not expressed (B), whereas endothelial damage was shown by leakage of vWF toward surrounding tumor tissue (C, arrows). In the absence of endothelial EMAP-II expression (D), VEGFR-2 is expressed (E). All stains were counterstained with Harris' hematoxylin. Magnification, $\times 250$. 
type. Our results also show that in uveal melanoma, macrophages are abundantly present and colocalize with the extracellular matrix patterns containing laminin. ${ }^{5,41}$

In uveal melanoma containing loops and network extracellular matrix PAS-positive patterns, the monocyte chemoattractant protein EMAP-II was abundantly present in the cytoplasm of tumor cells and was accompanied by local macrophage accumulation. Additional analysis of uveal melanomas lacking these patterns showed that different levels of local EMAP-II staining within one tumor were present and that macrophages were preferentially located in areas with highest EMAP-II immunoreaction. These data support the hypothesis that tumor cells recruit local resident tissue macrophages and peripheral monocytes by release of EMAP-II. This hypothesis was corroborated by several previous studies on the expression of EMAP-II in tumor cells and the ability of EMAP-II to attract macrophages. ${ }^{14,15}$ Indeed, infiltration of the tumor may be facilitated by the loops and network patterns by their serving as a gateway. However, secondary changes including extensive retinal detachments, glaucoma, rubeosis, and intraocular hemorrhage may contribute to the influx of macrophages as well, because most of the lesions analyzed were large. Because VEGF-C expression did not colocalize with absence or accumulation of macrophages, our data suggest that VEGF-C does not play a substantial role in macrophage invasion in uveal melanoma.

EMAP-II is normally retained intracellularly and can be released and partially processed by several triggers, including apoptosis and hypoxia. ${ }^{17,20}$ Because apoptotic cells were hardly present in the tumor lesions, this does not seem to be a decisive factor in EMAP-II expression in uveal melanoma. Hypoxic tumor cells may express hypoxia inducible factor (HIF)$1^{8,42}$ and release vascular endothelial growth factor (VEGF)-A. In this respect, it is noteworthy that we observed no release of VEGF-A in these primary uveal melanoma lesions. ${ }^{13}$ However, to the best of our knowledge, expression of HIF-1 in uveal melanoma has not been reported thus far.

Purified recombinant mature EMAP-II protein activates endothelial cells and enhances the expression of adhesion molecules P- and E-selectin. ${ }^{14}$ These adhesion molecules mediate invasion of different types of leukocytes into the underlying tissue (for review, see Ref. 27). Macrophage infiltration is primarily mediated by ICAM- 1 and VCAM- 1 adhesion molecules, however. Remarkably, one of our first observations was that EMAP-II and ICAM-1 are coexpressed on tumor cells. This indicates that either both these molecules are upregulated by the same stimulus, or that EMAP-II expression induces ICAM-1 expression by an autocrine mechanism. Moreover, ICAM-1 was predominantly expressed on the vascular endothelium in those tumors that showed intense ubiquitous staining for EMAP-II. In tumors with focal EMAP-II immunoreaction, the endothelium was often negative for both EMAP-II and ICAM-1, suggesting that a certain threshold level of EMAP-II binding to endothelial cells is necessary for induction of ICAM-1. Functional experiments in cell cultures are necessary to confirm these findings. Because only four tumors expressed vascular cell adhesion molecule (VCAM) on their vessels, it is unlikely that this adhesion molecule is induced by EMAP-II expression and contributes to infiltration of monocytes. ${ }^{27}$

To elucidate further the role of endogenously produced EMAP-II in primary uveal melanoma, we evaluated additional parameters that have been associated with EMAP-II expression in the literature-that is, the induction of apoptosis and of angiogenesis. As stated earlier, EMAP-II is involved in macrophage influx in several types of carcinomas. This has been related to tumor progression ${ }^{9,10}$ and may be explained partly by macrophage-induced neovascularization ${ }^{43}$ (for review see Ref. 44). We hypothesized that in primary uveal melanoma, hypoxic tumor cells secrete EMAP-II, contributing to recruit- ment and infiltration of monocytes into the tumor tissue. Our results suggest that this sequence of events is not followed by angiogenesis: EMAP-II-positive endothelium did not express VEGFR-2 as a sign of endothelial activation, as occurs during angiogenesis. Rather, our data suggest extensive release or leakage of VWF out of the EMAP-II-positive endothelial cells into surrounding tumor tissue, indicating endothelial cell damage. These findings imply that EMAP-II, instead of triggering angiogenesis, rather induces endothelial cell death in vivo. In uveal melanoma therefore, EMAP-II appears to have an adverse effect on the neovasculature, supporting the results obtained by administering exogenous EMAP-II in previous studies. $^{14,18,45}$ It has been demonstrated that EMAP-II induces endothelial apoptosis ${ }^{18}$ after release by apoptotic (tumor) cells. However, in our study, no evident apoptosis of EMAP-II-positive endothelial or tumor cells was observed. The involvement of apoptosis, either as a trigger of EMAP-II induction or as a result of its formation, remains therefore questionable, at least in uveal melanoma. Our data support earlier results indicating that processing and release of EMAP-II is not restricted to apoptotic cells but may also occur in cells that ultimately die by ischemia and subsequent necrosis.

The question arises whether local administration of EMAP-II will induce extensive vascular damage that may lead to necrosis of the tumor. Previous data from our laboratory indicate that the PAS-positive loops and network patterns may be involved in tumor cell nutrition (Clarijs R, van Dijk M, van Kraats A, Ruiter DJ, de Waal RMW, manuscript submitted). Thus, by inhibition of angiogenesis through administration of EMAP-II, uveal melanoma may (partly) escape this intervention by formation of loops and network patterns. Local administration of EMAP-II may also lead to extensive influx of macrophages, which may trigger an immune response or, alternatively, result in release of growth factors necessary for tumor growth and subsequent metastasis. Thus, the effects of EMAP-II are complex, and the possible effects of intervention with expression of EMAP-II in uveal melanoma or other tumor types are yet unpredictable and therefore require extensive additional research.

Data on the clinical outcome of 16 of the patients were obtained, with only 1 patient found to have metastatic disease. This could be explained by the relatively short follow-up in the patients with primary uveal melanoma, because this tumor is notorious for metastasis after several years. Therefore, our study did not allow us to draw any conclusion on the role of EMAP-II expression in determining prognosis.

In summary, our data are in line with the following sequence of events: Tumor cells promote adhesion and invasion of macrophages through the release of EMAP-II. This process is mediated by EMAP-II-induced expression of ICAM-1 on endothelial cells. Infiltrating macrophages follow the PAS-positive extracellular matrix patterns. Binding of substantial amounts of EMAP-II to vascular endothelial cells may lead to endothelial damage, resulting in $\mathrm{vWF}$ release.

\section{References}

1. Folberg R, Pe'er J, Gruman LM, et al. The morphologic characteristics of tumor blood vessels as a marker of tumor progression in primary human uveal melanoma. Hum Pathol. 1992;23:12981305.

2. Folberg R, Rummelt V, Parys-van Ginderdeuren R, et al. The prognostic value of tumor blood vessel morphology in primary uveal melanoma. Opbthalmology. 1993;100:1389-1398.

3. Warso MA, Maniotis AJ, Chen X, et al. Prognostic significance of periodic acid-Schiff-positive patterns in primary cutaneous melanoma. Clin Cancer Res. 2001;7:473-477. 
4. Makitie T, Summanen P, Tarkkanen A, Kivela T. Microvascular loops and networks as prognostic indicators in choroidal and ciliary body melanomas. J Natl Cancer Inst. 2000;91:359-367.

5. Clarijs R, Otte-Höller I, Ruiter DJ, de Waal RM. Presence of a fluid-conducting meshwork in xenografted cutaneous and primary human uveal melanoma. Invest Ophthalmol Vis Sci. 2002;43:912918.

6. Mäkitie T, Summanen P, Tarkkanen A, Kivela T. Tumor-infiltrating macrophages $(\mathrm{cd} 68(+)$ cells) and prognosis in malignant uveal melanoma. Invest Ophthalmol Vis Sci. 2001;42:1414-1421.

7. Mäkitie T, Tarkkanen A, Kivela T. Comparative immunohistochemical oestrogen receptor analysis in primary and metastatic uveal melanoma. Graefes Arch Clin Exp Ophthalmol. 1998;236:415419.

8. Bingle L, Brown NJ, Lewis CE. The role of tumour-associated macrophages in tumour progression: implications for new anticancer therapies. J Pathol. 2002;196:254-265.

9. Leek RD, Lewis CE, Whitehouse R, Greenall M, Clarke J, Harris AL. Association of macrophage infiltration with angiogenesis and prognosis in invasive breast carcinoma. Cancer Res. 1996;56:46254629.

10. Takanami I, Takeuchi K, Kodaira S. Tumor-associated macrophage infiltration in pulmonary adenocarcinoma: association with angiogenesis and poor prognosis. Oncology. 1999;57:138-142.

11. Vacca A, Ribatti D, Ruco L, Giacchetta F, et al. Angiogenesis extent and macrophage density increase simultaneously with pathological progression in B-cell non-Hodgkin's lymphomas. BrJ Cancer. 1999;79:965-970.

12. Skobe M, Hamberg LM, Hawighorst T, et al. Concurrent induction of lymphangiogenesis, angiogenesis, and macrophage recruitment by vascular endothelial growth factor-C in melanoma. Am J Pathol. 2001;159:893-903.

13. Clarijs R, Schalkwijk L, Ruiter DJ, de Waal RM. Lack of lymphangiogenesis despite coexpression of VEGF-C and its receptor Flt- 4 in primary uveal melanoma. Invest Ophthalmol Vis Sci. 2001;42: $1422-1428$.

14. Kao J, Houck K, Fan Y, et al. Characterization of a novel tumorderived cytokine: endothelial-monocyte activating polypeptide II. J Biol Chem. 1994;269:25106-25119.

15. Kao J, Ryan J, Brett G, et al. Endothelial monocyte-activating polypeptide II: a novel tumor-derived polypeptide that activates host-response mechanisms. J Biol Chem. 1992;267:20239-20247.

16. Schwarz M, Lee M, Zhang F, et al. EMAP II: a modulator of neovascularization in the developing lung. Am J Physiol. 1999; 276:L365-L375.

17. Knies UE, Behrensdorf HA, Mitchell CA, et al. Regulation of endothelial monocyte-activating polypeptide II release by apoptosis. Proc Natl Acad Sci USA. 1998;95:12322-12327.

18. Schwarz MA, Kandel J, Brett J, et al. Endothelial-monocyte activating polypeptide II: a novel antitumor cytokine that suppresses primary and metastatic tumor growth and induces apoptosis in growing endothelial cells. J Exp Med. 1999;190:341-354.

19. Marvin MR, Libutti SK, Kayton M, et al. A novel tumor-derived mediator that sensitizes cytokine-resistant tumors to tumor necrosis factor. J Surg Res. 1996;63:248-255.

20. Barnett G, Jakobsen AM, Tas M, et al. Prostate adenocarcinoma cells release the novel proinflammatory polypeptide EMAP-II in response to stress. Cancer Res. 2000;60:2850-2857.

21. Fauser S, Nguyen TD, Bekure K, Schluesener HJ, Meyermann R Differential activation of microglial cells in local and remote areas of IRBP1169-1191-induced rat uveitis. Acta Neuropathol (Berl) 2001; 101:565-571.

22. Zhang F, Schwarz MA. Temporo-spatial distribution of endothelialmonocyte activating polypeptide II: an anti-angiogenic protein, in the mouse embryo. Dev Dyn. 2000;218:490 - 498.

23. Knies UE, Kroger S, Clauss M. Expression of EMAP II in the developing and adult mouse. Apoptosis. 2000;5:141-151.

24. Schluesener HJ, Seid K, Deininger M, Schwab J. Transient in vivo activation of rat brain macrophages/microglial cells and astrocytes by immunostimulatory multiple CpG oligonucleotides. J Neuroimmunol. 2001;113:89-94.
25. Schluesener HJ, Seid K, Zhao Y, Meyermann R. Localization of endothelial-monocyte-activating polypeptide II (EMAP II), a novel proinflammatory cytokine, to lesions of experimental autoimmune encephalomyelitis, neuritis and uveitis: expression by monocytes and activated microglial cells. Glia. 1997;20:365-372.

26. Tas MPR, Murray C. Endothelial-monocyte-activating polypeptide II. Int J Biochem Cell Biol. 1996;28:837-841.

27. Bevilacqua MP. Endothelial-leukocyte adhesion molecules. Annu Rev Immunol. 1993;11:767-804.

28. Chang SY, Park SG, Kim S, Kang CY. Interaction of the C-terminal domain of p43 and the alpha subunit of ATP synthase: its functional implication in endothelial cell proliferation. J Biol Chem. 2002;277:8388-8394

29. Wu PC, Alexander HR, Huang J, et al. In vivo sensitivity of human melanoma to tumor necrosis factor (TNF)-alpha is determined by tumor production of the novel cytokine endothelial-monocyte activating polypeptide II (EMAPII). Cancer Res. 1999;59:205-212.

30. Gnant MF, Berger AC, Huang J, et al. Sensitization of tumor necrosis factor alpha-resistant human melanoma by tumor-specific in vivo transfer of the gene encoding endothelial monocyte-activating polypeptide II using recombinant vaccinia virus. Cancer Res. 1999;59:4668-4674

31. Ortega N, Jonca F, Vincent $S$, et al. Systemic activation of the vascular endothelial growth factor receptor KDR/flk-1 selectively triggers endothelial cells with an angiogenic phenotype. Am J Pathol. 1997;151:1215-1224.

32. Rose M, Page C, Hengstenberg C, Yacoub M. Immunocytochemical markers of activation in cardiac transplant rejection. Eur Heart J. 1991;12(suppl D):147-150.

33. Foss AJ, Alexander RA, Hungerford JL, et al. Reassessment of the PAS patterns in uveal melanoma. BrJ Ophthalmol. 1997;81:240246.

34. Daemen MARC, van 't Veer C, Denecker G, et al. Inhibition of apoptosis induced by ischemia-reperfusion prevents inflammation. J Clin Invest. 1999;104:541-549.

35. Verbeek MM, Otte-Holler I, Westphal JR, et al. Accumulation of intercellular adhesion molecule-1 in senile plaques in brain tissue of patients with Alzheimer's disease. Am J Pathol. 1994;144:104116.

36. Kerstens HM, Poddighe PJ, Hanselaar AG. A novel in situ hybridization signal amplification method based on the deposition of biotinylated tyramine. J Histochem Cytochem. 1995;43:347-352.

37. Mäkitie T, Summanen P, Tarkkanen A, Kivela T. Microvascular density in predicting survival of patients with choroidal and ciliary body melanoma. Invest Ophthalmol Vis Sci. 1999;40:2471-2480.

38. Facchetti F, Bertalot G, Grigolato PG. KP1 (CD 68) staining of malignant melanomas. Histopathology. 1991;19:141-145.

39. Maniotis AJ, Folberg R, Hess A, et al. Vascular channel formation by human melanoma cells in vivo and in vitro: vasculogenic mimicry. Am J Pathol. 1999;155:739-752.

40. Bittner M, Meltzer P, Chen Y, et al. Molecular classification of cutaneous malignant melanoma by gene expression profiling. $\mathrm{Na}$ ture. 2000;406:536-540.

41. Seftor RE, Seftor EA, Koshikawa N, et al. Cooperative interactions of laminin 5 gamma 2 chain, matrix metalloproteinase-2, and membrane type-1-matrix/metalloproteinase are required for mimicry of embryonic vasculogenesis by aggressive melanoma. Cancer Res. 2001;61:6322-6327.

42. Wang GL, Semenza GL. General involvement of hypoxia-inducible factor 1 in transcriptional response to hypoxia. Proc Natl Acad Sci USA. 1993;90:4304-4308.

43. Polverini PJ, Leibovich SJ. Induction of neovascularization in vivo and endothelial proliferation in vitro by tumor-associated macrophages. Lab Invest. 1984;51:635-642.

44. Sunderkotter C, Steinbrink K, Goebeler M, Bhardwaj R, Sorg C. Macrophages and angiogenesis. J Leukoc Biol. 1994;55:410 - 422.

45. Kao J, Fan YG, Haehnel I, et al. A peptide derived from the amino terminus of endothelial-monocyte-activating polypeptide II modulates mononuclear and polymorphonuclear leukocyte functions, defines an apparently novel cellular interaction site, and induces an acute inflammatory response. J Biol Chem. 1994;269:97749782 . 